\title{
A New Scheduling Scheme for Resilient Packet Ring Networks with Single Transit Buffer *
}

\author{
Jian Zhu Ashraf Matrawy Ioannis Lambadaris \\ \{jianzhu, amatrawy,ioannis\}@sce.carleton.ca \\ Department of Systems and Computer Engineering \\ Carleton University, Ottawa, Ontario, Canada \\ Mohsen Ashourian \\ mohsena@iaumajlesi.ac.ir \\ Islamic Azad University of Iran, Majlesi Branch, Isfahan, Iran
}

\begin{abstract}
Resilient Packet Ring (RPR) technology known as IEEE 802.17 standard is an emerging network architecture and technology designed to meet the requirements of a packet-based metropolitan area network. The medium access control architecture of RPR network can have single transit buffer (STB), or dual transit buffer (DTB). STB has lower implementation complexity. But the weakness of single transit buffer architecture is that high priority traffic trying to access the ring will be delayed by any low priority traffic already transiting on the node. To improve the quality of service for high priority traffic transmission, we propose a new RPR scheduling scheme which uses Deficit Round-Robin (DRR) algorithm to alternately select packets from the single transit buffer and the high priority transmit buffer. If there is no packet in these two buffers, low priority transmit buffer is then served. Simulation results show certain improvement on overall delay and delay jitter performance of RPR networks with single transit buffer by using our scheme.
\end{abstract}

Keywords: Resilient Packet Ring, Scheduling, Deficit Round-Robin.

\section{Introduction}

Resilient Packet Ring (RPR)[1][2], an IEEE 802.17 standard, is ring network based on a highly scaleable and resilient technology for the efficient transfer of packetbased traffic across a network. RPR networks offer multiple performance benefits: such as spatial reuse, bandwidth efficiency, ease of management, resilience and scalability. These advantages make RPR a very attractive alternative for high speed MANs and LANs.

RPR has two ring architectures: Single Transit Buffer (STB), and Dual Transit Buffer (DTB) [1][2][3][7]. Fig. 1 shows the Single Transit Buffer configuration. For both STB and DTB architectures, a high priority and low priority transmit buffer exist at the client side. In the STB architecture, a single transit buffer amalgamates Low Priority (LP) and High Priority (HP) traffic from the transmit buffer into one mixed traffic buffer on the ring. In the DTB architecture, two transit buffers exist for LP and HP traffic. When the traffic arrives from the ring, it is either dropped

*This work is supported by CITO (Communications and Information Technology Ontario) or destined from that node, or placed in the high priority or low priority transit buffer, according to the traffic classification.

Priority Queue (PQ)[4] algorithm is used as the scheduling scheme for RPR. The scheduler handles a specific queue on the basis that all the higher priority queues have been served and emptied. This approach ensures low delay for higher priority classes of traffic. However, it often leads to the starvation of lower priority queues. For the single transit buffer architecture, traffic on the ring, which is a mixture of HP and LP transit traffic, has higher priority over the transmit HP traffic. This could cause the LP traffic on the ring block the transmit HP traffic from gaining access onto the ring, which is not suit for the Quality of service $(\mathrm{QoS})$ requirements of HP traffic in terms of delay and delay jitter.

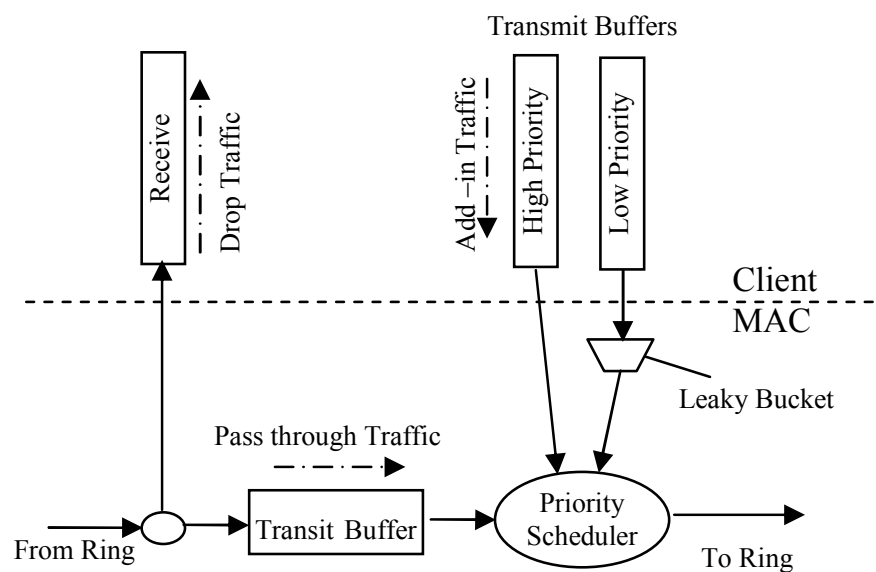

Fig. 1. The MAC architecture in RPR with STB

To improve the QoS of HP traffic transmission, we propose a new scheduling scheme for RPR networks with STB. In our scheme, traffic is classified as two levels. Single transit buffer traffic and HP transmit buffer traffic are deemed as first level traffic. We implement Deficit RoundRobin (DRR) algorithm [5] to alternately select packets from these two buffers. Traffic from LP transmit buffer is deemed as second level traffic. Only when there is no first 
level traffic in the buffer, second level traffic can be served by the scheduler.

Since DRR only requires $O(1)$ work to process a packet, our scheme does not add extra complexity to the original architecture. Simulation results show certain improvement on overall delay and delay jitter performance of RPR networks with single transit buffer by using our scheme.

The paper is organized as follows: Section II describes the RPR technology. An overview on DRR is provided in Section III. Section IV contains our proposed scheduling scheme. Simulation results and analysis are presented in section V. Conclusions will be drawn in Section VI.

\section{Resilient Packet Ring (RPR)}

RPR connects nodes into a point-to-point, full duplex ring topology. Unlike token ring, in RPR several packets might be on their way at the same time, provided the packets use different segments of the ring. This is called spatial reuse. A subnet that connects all the nodes and moves traffic in one direction around the ring is called a ringlet. RPR spatially reuse the ring bandwidth by letting the destinations strip the packets. Hence one packet may flow on one segment of a ringlet while other packet flows on another part of the same ringlet at the same time. Each node is connected to two ringlets, and has a full duplex connection to the outside. Each ringlet interface includes transmit, transit and drop buffers. Packets on their way into the ring are stored in the transmit buffer, while packets that are stripped form the ring are stored in the drop buffer. Packets that are traveling on the ring are stored in each nodes' transit buffer to be processed.

Access to the ring is controlled by a fairness or media access control (MAC) algorithm. A control system with feedback to upstream stations is used in order to avoid starvation and achieve fair access to the ring. The basis of such a control mechanism is flow control packets that a node sends upstream when it does not get its fair share of the bandwidth (it is about to be starved). Such a packet contains the starving stations current end rate. When the upstream nodes receive flow control packets they adjust their leaky bucket leak rates to the fair rate advertised in the flow control packets. When a node does not receive any more flow packets, it gradually increases its send rate again. Fair rate calculations are made every Sample Period, which is typically set to $100 \mu \mathrm{s}$. It is important to note that only low priority traffic is subjected to the fairness algorithm.

Each node of RPR has a high priority and low priority transmit buffer at the client side. Real time data such as video and voice can be classified as high priority traffic, which suffers more from packet loss and delay. Low priority traffic can be attributed to other non-essential or noncritical data, which may suffers from delay and packet loss, but still meets the application requirements. The tran- sit buffers can be configured such that it can have one transit buffer, or two transit buffers.

In the single transit buffer architecture, a transit buffer combines mixed low priority and high priority traffic. The traffic in that buffer has the utmost priority. The advantage of this configuration is that it simplifies the hardware implementation since it is less complex. However the traffic on the ring, which is a mixture of high priority and low priority traffic, can block the transmitted high priority and low priority traffic waiting to gain access onto the ring. The MAC scheduler sends traffic in the following order:

1) Packets sent from the mixed transit buffer.

2) Packets sent from the high priority transmit buffer.

3) Packets sent from the low priority transmit buffer.

\section{Deficit Round-Robin (DRR) Algorithm}

The round-robin schedulers divide the time line into allocation cycles and allocate each connection a fraction of the available bandwidth in each cycle. The main attraction of this class of schedulers is their simplicity. The DRR algorithm is an extension of the round-robin algorithm. Instead of processing exactly one packet from each queue, DRR associates a quantum of service for each queue.

The DRR scheduler maintains a linked list of the active flows, called the Active List. At the start of an active period of a flow, the flow is added to the tail of the Active List. A flow is said to be active during a certain time interval, if it always has packets awaiting service during this interval. A round is defined as one round-robin iteration during which the DRR scheduler serves all the flows that are present in the Active List at the outset of the round. Each active flow is assigned a quantum by the DRR scheduler. Suppose that transmission rate of an output link is $r$. Let $n$ be the total number of flows and let $p_{i}$ be the reserved rate for flow $i$. Let $p_{\min }$ be the lowest of these reserved rates. Note that since all the flows share the same output link, a necessary constraint is that the sum of the reserved rates be no more than the transmission rate of the output link. In order that each flow receives service proportional to its guaranteed service rate, the DRR scheduler assigns a weight to each flow. The weight assigned to flow is given by

$$
\mathrm{w}_{\mathrm{i}}=\mathrm{p}_{\mathrm{i}} / \mathrm{p}_{\min }
$$

Note that $w_{i} \geq 1$. Let $Q_{i}$ represent the quantum assigned to flow $i$ and let $\mathrm{Q}_{\min }$ be the quantum assigned to the flow with the lowest reserved rate. The quantum assigned to flow $\mathrm{i}, \mathrm{Q}_{\mathrm{i}}$ is given by $\mathrm{w}_{\mathrm{i}}{ }^{*} \mathrm{Q}_{\min }$. Thus, the quanta assigned to the flows are in proportion of their reserved rates. In order that the work complexity of the DRR scheduler is $\mathrm{O}(1)$, it is necessary that $\mathrm{Q}_{\min }$ should be greater than or equal to the size of the largest packet that may potentially arrive during the execution of the scheduler. Note that during some service opportunity, a flow may not be able to transmit a packet because doing so would cause the flow to 
exceed its allocated quantum. The scheduler maintains a per-flow state, the deficit count, which records the difference between the amount of data actually sent thus far, and the amount that should have been sent. This deficit is added to the value of the quantum in the next round, as the amount of data the scheduler should try to schedule in the next round. Thus, a flow that received very little service in a certain round is given an opportunity to receive more service in the next round.

DRR can be easily adapted for guaranteed-rate service scheduling and is the most efficient scheduling algorithm for high speed network for its $\mathrm{O}(1)$ complexity, and low operational cost $[5,6]$.

\section{The Proposed Scheduling Scheme in RPR}

In Resilient Packet Ring standard, priority queue is recommended as scheduling algorithm. For the single transit buffer architecture, traffic on the ring has higher priority over transmit traffic. Even the low priority transittraffic is served before high priority transmit-traffic. During congestion interval, overwhelming low priority transit traffic can block the transmission of high priority traffic waiting to gain access onto the ring. This is unfair for the local transmit high priority traffic. It also degrades the total delay and delay jitter performance for the high priority traffic.

To improve the Qos of high priority traffic, we substitute part of the original priority queue scheduling algorithm with deficit round-robin algorithm. We use deficit round-robin scheduling between the transit buffer and transmit high priority buffer. Only when there is no packet in both the transit buffer and transmit high priority buffer, the transmit low priority buffer is served by the scheduler. Fig. 2 shows the MAC architecture of our scheme. Using DRR in our scheme can guarantee the regularly access of the high priority transmit traffic to the ring and it only requires $\mathrm{O}(1)$ work to process a packet. Therefore, it does not add extra complexity to the original architecture.

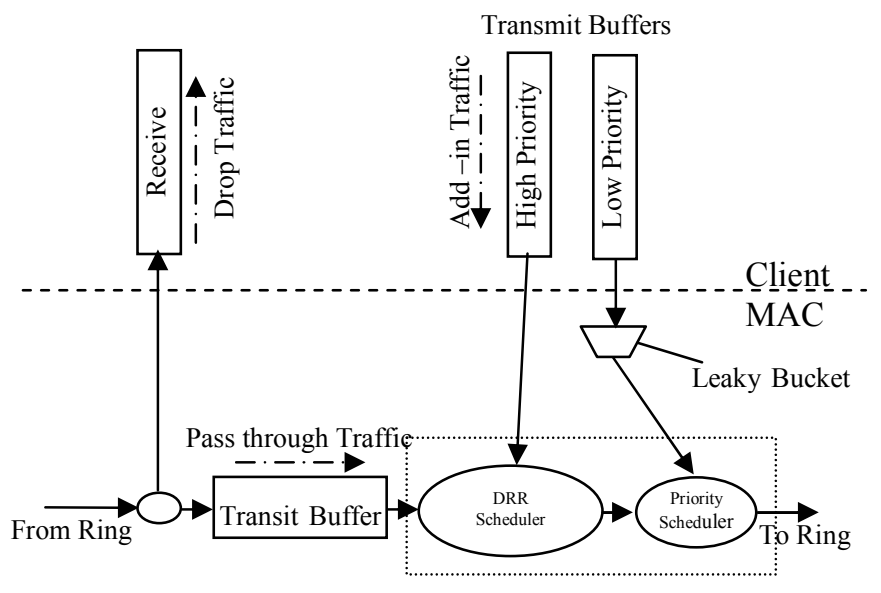

Fig. 2. The MAC architecture in the proposed scheme.

\section{Simulation Results and Analysis}

We simulate a 10-node ring network in OPNET modeler. Fig. 3 shows the RPR network topology with Hub scenario in our simulation. Four nodes $(6,7,8,9)$ send packets on the outer ring to the terminal node 0 , the Hub. Observations are made in the Hub. Both schemes use the RPR recommended fairness mechanism for single transit buffer [1,2]. Table 1 shows the simulation parameters. The three packet sizes emulate real traffic profile.

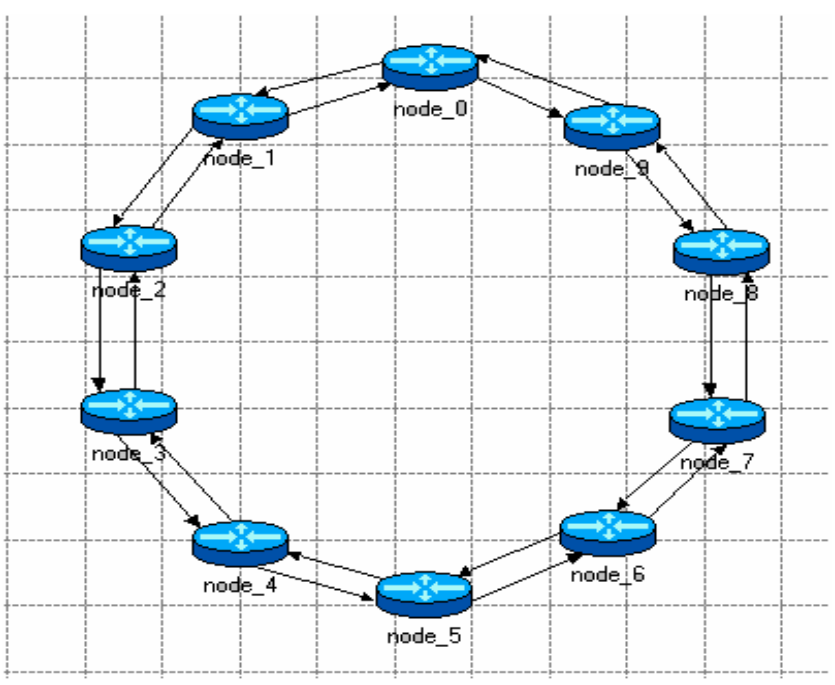

Fig. 3. RPR network topology used in the simulation.

Table 1. Simulation Parameters

\begin{tabular}{|l|l|}
\hline Parameters & Value \\
\hline Link Capacity & $100 \mathrm{Mbit} / \mathrm{s}$ \\
\hline Fairness Sample Period & $100 \mu \mathrm{s}$ \\
\hline Transit Queues Size & $1 \mathrm{Mega}$ bits \\
\hline & $60 \%: 64$ byte \\
Packet Sizes & $20 \%: 512$ byte \\
& $20 \%: 1518$ byte \\
\hline Traffic Generated in & HP:10 Mbits/s \\
Each Node & LP: $23 \mathrm{Mbits} / \mathrm{s}$ \\
\hline
\end{tabular}

The DRR scheduler assigns a weight to transmit high priority flow proportional to its guaranteed high priority transmit traffic service rate. The remaining portion is assigned to the transit buffer. For example if the guaranteed high priority transmits traffic rate of node 6 is $10 \mathrm{M}$ bits/s, and the total link capacity is $100 \mathrm{M}$ bits/s, then we assign weight 1 to transmit high priority traffic buffer of node 6 , and weight 9 to its transit buffer. In this way, the service of transmit high priority buffer can always be guaranteed. 


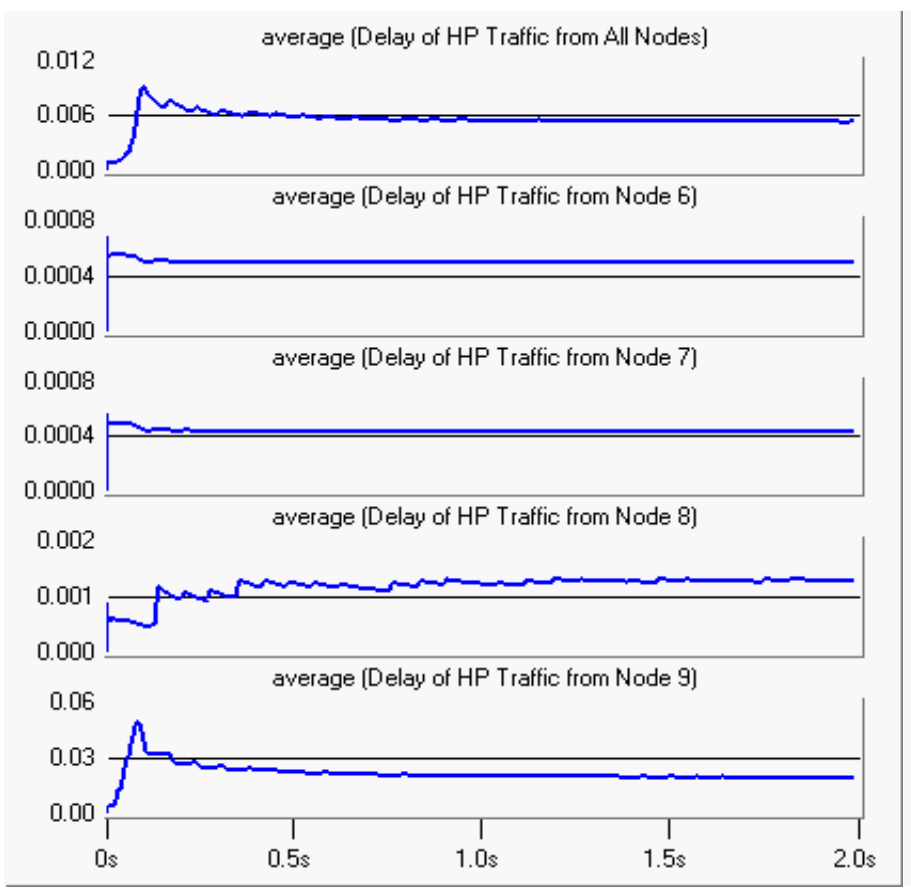

Fig. 4. The delay of HP traffic using original RPR scheduling

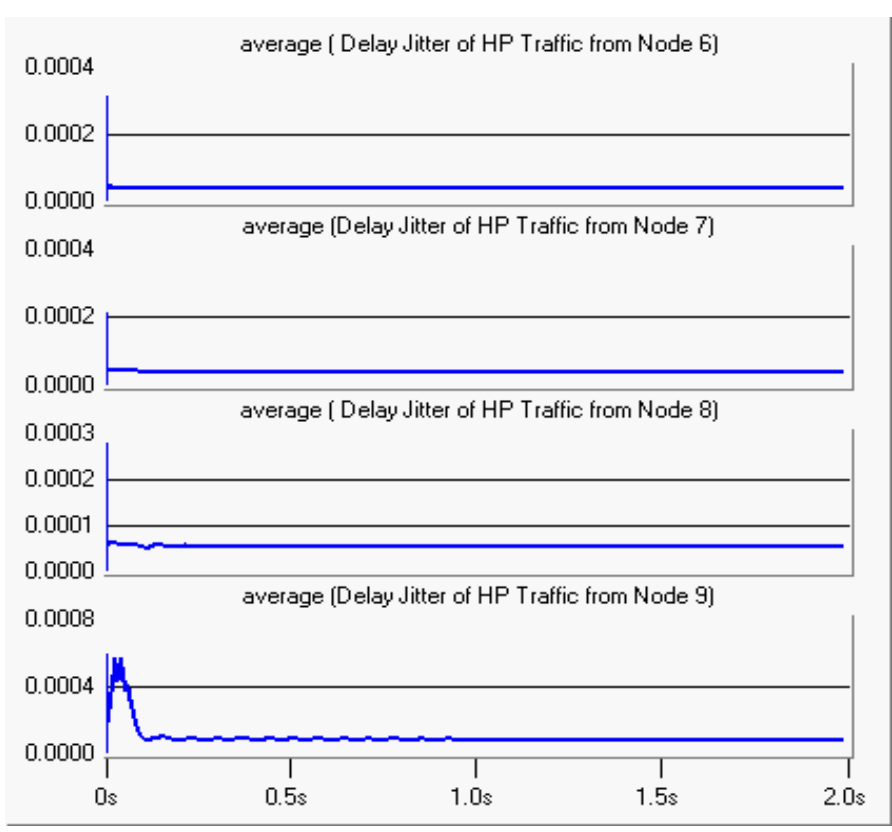

Fig. 6. The delay jitter of HP traffic using original RPR scheduling

The generated traffic rate from each node is 33 Mbits/s, that has $10 \mathrm{Mbits} / \mathrm{s}$ high priority traffic, and 23 $\mathrm{Mbits} / \mathrm{s}$ low priority traffic. This results to congestion at node 9. If we use original RPR scheduling scheme, the transit buffer will be emptied first. High priority transmit

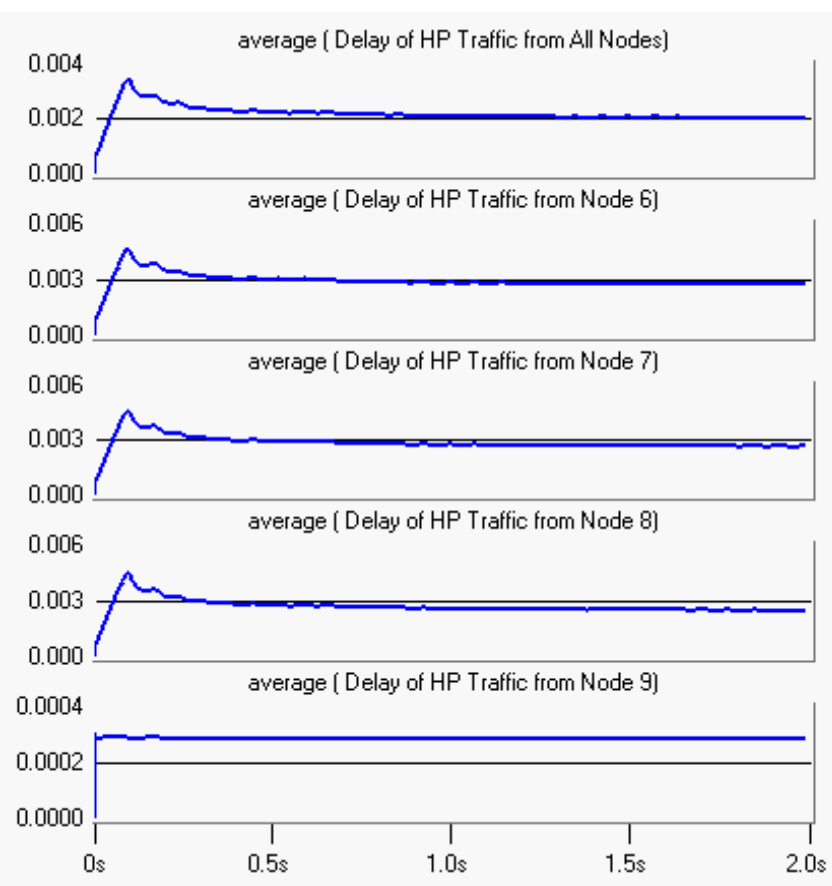

Fig. 5. The delay of HP traffic using proposed RPR scheduling

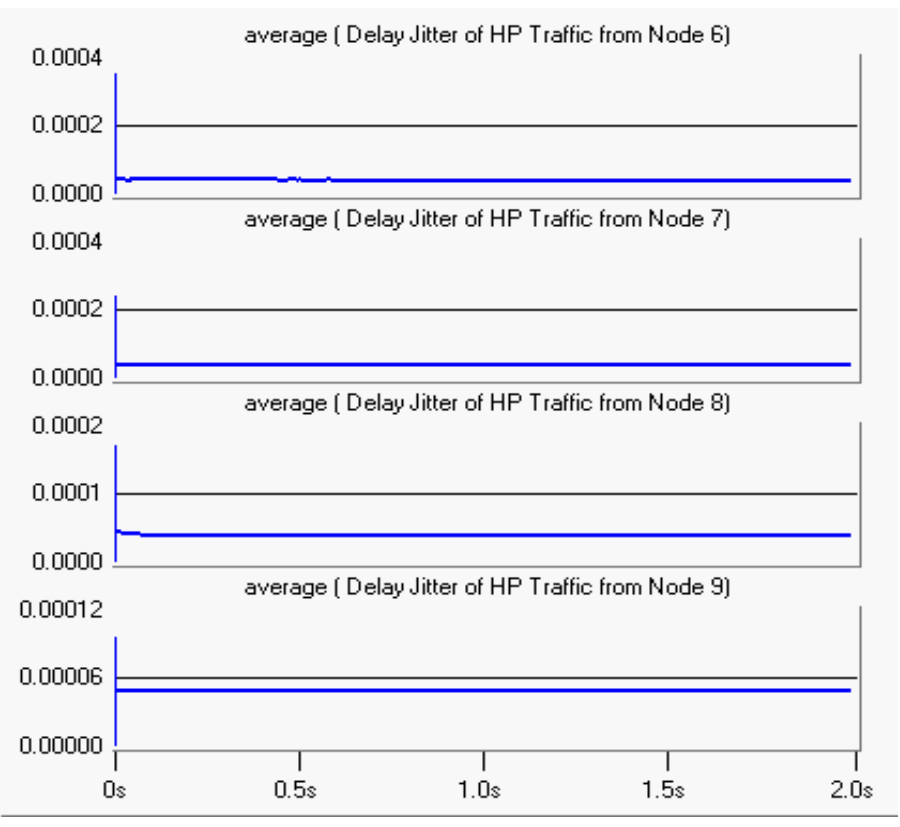

Fig. 7. The delay jitter of HP traffic using proposed RPR scheduling

traffic of node 9 is always queued for serving; even its priority is lower than the low priority traffic from node 6 , 7,8 . Also the delay jitter of node 9 high priority transmit traffic depends on the congestion situation of the single transit buffer. 
We compare the proposed scheduling scheme performance in terms of delay and delay jitter with the original RPR scheduling scheme. Fig. 4 and Fig.5 show the end-to-end delay of high priority traffic which demonstrates an average decrease of around one hundred times for the congested node (Node 9). We also achieve better end-to-end delay for the overall high priority traffic using our proposed scheduling scheme.

The average delay of high priority traffic from the congested node (Node 9) in the proposed scheme always reaches to its stable situation (with a maximum variation of $10 \%$ ) in less than 0.1 second. This period is above 0.25 second in the original RPR scheduling scheme. Regarding to the high priority traffic from other nodes, the time to reach steady is the same for both schemes.

Fig. 6 and Fig. 7 show the delay jitter of the original system and the proposed one for the high priority traffic. We can see that in all the nodes the delay jitter reach to stable value much faster in our proposed scheme. The improvement is especially more clear for the node 9 (the congested node). Despite the congestion in node 9, the high priority traffic in it still has the chance to pass onto the ring.

By comparing the occupied buffer size of Node 9 using two different schemes, We find that our proposed scheme needs more transit buffer size. In our simulation, the proposed scheduling scheme needs $25 \%$ of the 1 Megabits total transit buffer, while the original scheme occupied $1 \%$ of the total transit buffer. The reason is that in original RPR scheduling, the transit buffer always has the highest priority and emptied immediately. However in the proposed scheduling scheme, DRR algorithm serve the transit traffic and high priority transmit traffic alternatively.

We also observe that throughput of high priority traffic in both schemes was $100 \%$.

\section{Conclusions}

In this paper, a new scheduling scheme for RPR which use Deficit Round-Robin scheduling algorithm to substitute part of its original priority queue algorithm is presented. We simulated the proposed scheme in an RPR network with 10 nodes with Hub scenario and compared the system performance in terms of delay and delay jitter. Simulations showed that our solution can always guarantee the fair access of transmit high priority buffer. The proposed scheme shows much lower end-to-end delay, and lower delay jitter. The proposed scheme can be further optimized for special types of real time traffic such as speech or video data.

References

[1]A. Mekkittikul et al. "Aladdin Proposal for IEEE Standard 802.17”, Draft 1.0, Nov. 2001.
[2]J. Kao et al. "Darwin Proposal for IEEE Standard 802.17", Draft 1.0, Jan. 2002.

[3]D. Tsiang, G. Suwala, "CISCO SRP MAC layer Protocol", Internet Engineering Task Force (IETF) Request for Comments 2892, August2000.

[4]Kleinrock, L: Queuing Systems. First Volume; John Wiley \& Sons, New York ,1975.

[5]M. Shreedhar and G. Varghese, "Efficient fair queuing using deficit round-robin," IEEE/ACM Trans. Networking, Vol. 4, pp. 375-385, June 1996.

[6]D. Saha, S. Mukherjee, and S. K. Tripathi, “ Carry-Over Round-Robin: a simple cell scheduling mechanism for ATM networks," IEEE/ACM Trans. Networking, Vol.6, No.6, pp. 779-796, Sep. 1998.

[7] M. J. Francisco, F. Yuan, C. Huang, and H. Peng, "A Comparison of Two Buffer Insertion Ring Architectures with Fairness Algorithms," in Proceedings of $I C C^{\prime} 03$, Anchorage, May, 2003. 